\title{
A Review and Current Update on Sentinel Lymph Node Biopsy of Breast Cancer
}

\author{
Stacia Novia Marta ${ }^{1 *}$, Nyoman Dwi Aussi Hary Mastika ${ }^{1}$, Hendry $\operatorname{Irawan}^{2}$ \\ ${ }^{1}$ Department of Surgery, Bali Mandara General Hospital, Denpasar, Indonesia; ${ }^{2}$ Department of Surgery, Division of Surgical \\ Oncology, Faculty of Medicine, Udayana University, Sanglah General Hospital, Denpasar, Indonesia
}

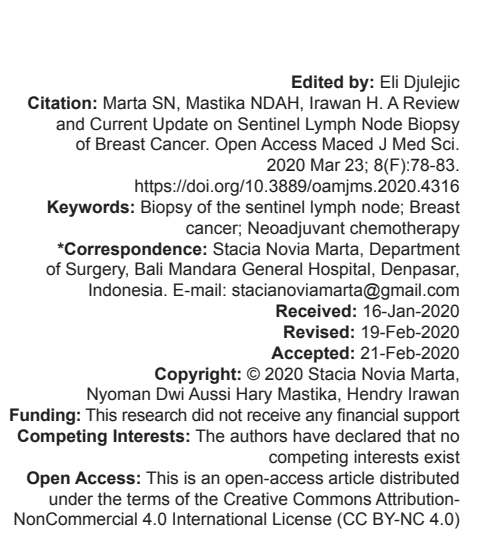

\section{Introduction}

Malignant tumor of the breast is currently accepted as a non-communicable disease burden all over the world. Data from cancer facts and figure 2018 [1] by the American Cancer Society reveals that about 1.7 million new cases will be diagnosed in 2018. It has also become the number one estimated new cases of cancer in women expected to be 266,120 . The death rate in a female caused by this particular cancer had progressively declined year by year and currently stands at $14 \%$. This achievement is considered an improvement attributed to early detection and treatment [2]. Experimental studies have been conducted to improve furthermore the screening and therapy technique of breast cancer to improve early detection and life aspects of patients with this particular disease, therefore, preventing complications and concomitantly elevating the 5 years survival rate.

Sentinel lymph node (SLN) is characterized by its properties as the foremost node by which of a lymphatic vessel where the above will receive the drainage of a specific anatomical area that has the properties of an immune system through which it is responsible for that particular area [2]. The condition of the nodes within the axilla is thought to be a significant predicting feature in the early stages of breast malignancies; thus, SLN biopsy (SLNB) has evolved to be the standard management in determining advanced metastases to the lymphatic node vessel. SLNB has now taken an important role thus has replaced axillary lymph node dissection (ALND) as the standard technique for staging in those with clinically node-negative malignancy of the breast. Mapping of the lymphatic nodes to reveal the sentinel node was achieved in 1992 using blue dye by Donald Morton for cutaneous melanoma. The procedure was initially used to discover the spread through the sentinel node in those suffering from melanoma in the interest to those which would have earned beneficial properties from an immediate dissection of a regional node [3]. Thus afterward, in 1993, Krag et al. have mapped these nodes by injection of a radioactive contrast utilizing gamma probes in the management of malignancy of the breast [4]. As for now, SLNB has revolutionized surgical treatments of early malignancy and has become the standard of care over axillary node dissection for staging grounds and or purpose in a negative node disease because it is precise while having a lesser extent of morbidity and has the properties of being non-invasive. Ongoing studies are currently being done to further explore the advantages and utility of the biopsy of the SLN in breast malignancies [5]. 
This article is committed to reviewing the evolution, current status, and function of SLNB in malignancies of the breast.

\section{SLN in Malignancies of the Female Breast}

SLN is by definition the foremost node which drains a tumor which is often more than one. In breast cancer, the condition of the node of the axilla is currently known as the ultimate prognosticator of disease-free and overall survival rate. Furthermore, it functions as a guide in determining the options of local and systemic adjuvant treatment strategies. The involvement of the axillary node has already been recognized as a marker of bad outcome where the 5-year survival rate which will have been decreased by approximately 28-40\% [6]. It should be stressed that surgery (axillary node) is recognized as an instrument for staging and control an area of a specific region to maximize the survival rate in these patients. To establish a definitive diagnosis of axillary metastasis, this will further require excision and histological examination of axillary lymph nodes.

The thorough processes taken in the evaluation of axillary node conditions involves the routine application of surgical staging where tissues (lymph) were removed between the vein of the axilla in the superior area, the muscle of the serratus anterior in the medial area, and the muscle of the latissimus dorsi within the lateral area. In the management of breast cancer ALND is currently well known as the ultimate standard (gold standard) for staging. It is accurate and sensitive but on the other hand, still has the downside of higher morbidities like longer operating time, pathology costs and several complications known for effects of formation of seroma, axilla sensory deprivation, edema of the arm and limitation of movement. Thus it renders a dilemmatic condition especially in those whose nodes are clinically negative, i.e., cNO because most of them will have a pathological free node ( $p N 0)$ and will be more exposed to invasive surgery, which leads to the development of higher morbidity outcome. All of the above mentioned will affect the quality of life in these patients for axillary control and survival which remain questionable. For this reason, several studies have been done to explore a less invasive tool for staging females with breast cancer.

After the introduction of SLNB, the morbidity for $\mathrm{pN} 0$ patients has been dramatically reduced. The results of the study Z0011 show that there was no differentiation between patients undergoing SLNB and axillary dissection after 10 years [7]. However, some considerations will have to be made by surgeons in $\mathrm{pN}+$ patients, whether opting for ALND, which stands by the guideline [8] or removal of the SLN.

The total lymph nodes to be removed will vary from patient to patient. In general, ALND is defined as the removal of 10 or more nodes. The German S3 guideline states that ten nodes are the lowest amount to take out for the pathological report [8]. Recent studies show that there is no survival benefit of the procedure because the removal of fewer than 4 nodes can already be used for an accurate diagnosis [9].

\section{The Evolution of SLNB}

The SLNB has been accepted and well known of its feature of less invasive alternative and has now replaced the full ALND for those who underwent primary breast operation in cNO and in the early stages of breast malignancies before breast-conserving management.

The foremost SLNB was performed in 1951 by Gould [10] during parotidectomy, and further technique was adopted for other diseases by Cabanas [11] in penile cancer and by Morton et al. [12] in a patient with cutaneous melanoma. By the next few years, this technique was adopted in the management of breast cancer in 1994 by Guliano et al. [13] at John Wayne Cancer Institute. It was known for their ability to identify the status of the nodes in the range of $95 \%$ compared with the dissection of the axilla by use of blue dye. This study further shows that it is attainable to identify sentinel node in breast malignancies and it is capable to accurately foresee the condition of the entire axillary vessel. Different SLNB injection technique was done by Krag et al. [5] by utilizing radioisotopes in conjunction with the gamma probe localization.

Several studies compared ALND and SLNB to evaluate the sensitivity of detection rate and postoperative morbidity. Data have shown that there was less morbidity of the arm and shoulders such as edema, sensory, and mobility deprivation after SLNB compared to ALND which correlates with a higher quality of life. NSABP B-32 is well known as the largest trial to evaluate sentinel node biopsy and has conceded that disease-free survival, regional control, and total survival were equivalent between sentinel node biopsy only group and combination of sentinel node biopsy in addition to the dissection of the axilla group [14]. A randomized clinical trial by The ACOSOG Z0011 (Alliance) aimed to evaluate the outcome of the dissection of the axilla versus no dissection on 10 years overall survival in those with invasive malignancies of the breast and SN metastasis which has stated that SLN dissection only was not inferior to overall survival compared to patients that had been managed by dissection of nodes within the axilla [7]. At present, SLNB evolved to be the merit of care for the management (surgery) and evaluation of malignancies of the breast because of its accuracy, limited morbidities while also has the properties of limiting an invasive operation. 


\section{Figures of Sentinel Nodes Identified in Malignancies}

The SLNs are characterized because of its properties to be one of the first to receive drainage from a malignant growth bed. In the axillary bed, there could be more than one of these detected. The question was how many SLNs are necessary to take out for it to attain a tolerable accuracy rate in addition to low falsenegative rate (FNR) since those numbers are related to complications of the axilla which contributes to further morbidity. Several studies have suggested the number of SLN's that should be removed in SLNB to yield a low FNR and that four nodes were considered an acceptable threshold number and removal of more SLNs will not yield higher staging accuracy [9].

A prospective multicenter study at the University of Louisville was conducted by Anees et al. [15] to evaluate determining elements which are correlated to the harvest of SLN. This was based on the proposition that taking out of more than four nodes will have elevated the quality of being unhealthful without increasing staging precision nor advantage over complete dissection of the axilla. Samples were relatively large with 3882 subjects over 8-year period. Three hundred and thirty-six surgeons had done the procedures without any definitive protocol to do SLN biopsy. Tangible tumor, the familiarity of the surgeon, and injection technique were related to the amount of (number) "sentinel lymph nodes" taken out and less than four SLNs were considered adequate for a diagnostic tool. Another substantial study measured the number of SLNs taken to see the relation of disease (specific) survival and results showed positive results in those that had three SLNs taken out having better outcomes. It will become understaging if only one or two SLNs were taken. This study showed no remarkable benefit in survival for those with SLNB only compared to SLNB followed by ALND treatment. The only additional information retrieved was the number of nodes containing metastases, while, on the other hand, this prognostic data would not have change systemic therapy decisions and will further result in a significantly higher cost [16].

From several data and literature, the authors conclude that the removal of four nodes should be sufficient and overall data suggest that this concept will have higher accuracy and lower FNR.

\section{SLNB Technique}

In malignancy, SLN is generally found under the arm with a relatively small proportion found elsewhere in the lymphatic system (breast). If the SLN is affirmative, possibilities of a positive node upstream could be expected, whereas a negative SLN shows that the high probability of all upstream nodes is of the same. SLN biopsy is done by locating the and thus identifying it utilizing a (labeling) substance by an option of a radiotracer, blue pigment(dye) or in combination, which is administered around the tumor before mastectomy or lumpectomy. This marker will then travel to the same track of the nodes that malignant cells would manifest, rendering surgeons to decide the one or two nodes have the most probability to be positive, either evaluating it using color visualization or Geiger counter. Injection techniques differ between institutions, surgeon preferences, and skills [13], [17].

There are currently three common mapping schemes incorporating variable tracers SLNB through the utilization of radioisotope, blue coloring, and the combination of both [5], [18]. Surgeons will have to individualize their preferences to suit their practice. Some literature reveals the identification rate can be optimized while minimizing FNR by incorporating dual agents, especially for those with limited experience and in such instances where misidentification and false-negative rates are known to be higher. Several conditions which have shown to have higher FNR were prior breast surgery, neoadjuvant chemotherapy, and high BMI index [17], [19].

The current benchmark (gold standard) for SLNB is a dual-modality technique (radioisotope and blue coloring). The technique incorporates administration of a technetium-labeled nanocolloid and blue coloring interstitially into the breast on all sides of the tumor or periareolar region. After localizing itself in the lymphatic network, a scintillation counter is utilized to determine SLN which accepts the chief drainage from the tumor through a lymphatic network (vessel). The blue coloring will assist with localization after incision and nodes that are blue, radioactive, or both are recognized as SLN, thus taken out and examined [20], [21].

Despite low false-negative and high detection rate, this method has several disadvantages of using technetium 99 which has only 6 h of half-life, therefore, restricting scheduling of surgery. The limitation of the combined SLNB and allergic reaction paved the way for the evolution of another method. Recent studies have focused on the use of indocyanine green fluorescence and contrast-enhanced ultrasound (CEUS) using microbubbles or superparamagnetic iron oxide nanoparticles, but data and studies are currently inconclusive regarding the identification of any significant benefit of the previous dual technique in terms of SLN detection rate [22], [23].

\section{SLNB after Neoadjuvant Chemotherapy}

Neoadjuvant chemotherapy was at first introduced to locally downstage advanced malignancies 
to ease surgery which will result in the improvement of disease-free and increasing survival rate in a considerable percentage (20-40) of patients [24]. NAC nowadays is also used as initial therapy for early breast malignancies before breast preserving surgeries. Before NAC node-staging was done by dissection of the axillary lymph nodes during breast operations which were correlated with higher morbidity. SLNB is by definition a less aggressive approach to determine nodal staging and results in lesser morbidities. SLNB before NAC can precisely assess initial node condition circumventing the side effect of lymphatic scarring or uneven node tumor response but become more challenging than SLNB after NAC as an appealing approach as NAC could downstage node conditions in several patients. Otherwise, this will become important to show recurrence rate and to set up for further therapy. Before such strategies may be offered as routine several questions need to be answered in accordance to its safety validity, predictive value, and optimal timing of SLNB before NAC [25].

Several studies have been performed to determine sentinel biopsy before and after NAC. The advantage of performing sentinel node biopsy before giving neoadjuvant chemotherapy lies in the predetermination of nodal status which will ultimately affect the next regiment that the patient will receive depending on tumor size and biopsy result. This method will not further force the patient to further surgeries and may delay the timing of chemotherapy, thus presenting the most precise regional-staging for them [26].

Varying literature reveals that NAC may exhibit a number of unwanted effects on the accuracy of SLNB. The primary tumors and metastatic nodes which respond to chemotherapy produce reactive changes such as fibrosis that can influence drainage patterns. Irregular response in the elimination of axillary nodes which in turn may result in decreased sentinel node accuracy after NAC [25]. Several studies which registered patients with an SLNB biopsy before $\left(1^{\text {st }}\right)$ and after $\left(2^{\text {nd }}\right)$ NAC group revealed a significantly decreased SLN identification in the second group.

A prospective, multicenter cohort study had been conducted by Kuehn et al. [26] took place at 103 institutions in Germany and Austria. The (SENTinel NeoAdjuvant) study was aimed to assess an algorithm for the timing of a standardized SLNB in those who underwent NAC. The key endpoint of the study was the accuracy of SLNB after NAC, secondary endpoints is a comparison of the detection rate of SLNB before and after NAC, and also the FNR and detection rate of SNLB after taking out the SLN. In conclusion, SLNB is a well-founded diagnostic method before NAC, but this procedure has a lower detection rate and a higher FNR compared with SLNs performed before NAC.

Another multicenter prospective study was performed by Enokido et al. at eight centers in Japan [27]. This study, with a sample of 143 patients with breast malignancies with positive axillary nodes proven by FNAB cytology at the initial diagnosis (T1-T3N1M0), was done to determine the suitability of SLNB for patients with node-positive breast malignancies before NAC. In this study, SLNB was done by blue coloring and radiolabeled colloid agents to optimize the chance of SLN identification. In conclusion, the study of breast cancer patients who had ALN conversion from cytologydetermined positive to negative following NAC indicated that SLNB and ALND without SLNB had similar rates of axillary recurrence, disease-free survival, and overall survival. Therefore, SLNB may be acceptable after NAC for patients with cytology determined, node-metastasispositive breast cancer with a reasonable identification rate. Furthermore, in some patients, SLNB can help identify possible downstaging to a negative nodal stage, and since ALND is avoided, this may reduce arm motion morbidity and lymphedema. We suggest the use of SLN surgery as an alternative to ALND after NAC in patients with cytology determined axillary node metastasis positive breast cancer [27].

There were also several factors which contribute to sentinel node identification rate and falsenegative rate after neoadjuvant chemotherapy. Several studies point to have a low detection rate and a high FNR. Initial reports from a single institution showed inconsistency in sentinel node identification rates after neoadjuvant chemotherapy from $72 \%$ to $100 \%$ and FNRs of $0-33 \%$ [28]. The Ganglion sentinelle et chimiotherapie neoadjuvante study was a prospective study with conservative breast surgery, sentinel biopsy, and level I and II axillary lymphadenectomy after NAC for large operable breast malignancies [29]. This study was conducted from September 2003 until March 2007, with a total of 195 subjects prospectively included from 12 institutions. The study showed that the detection rate was $90 \%$, the FNR $11.5 \%$ and that subjects with no palpable axillary nodes (NO) before NAC had a better detection rate compared to patients with axillary suspicious nodes. This study finally concluded the practicality of sentinel node biopsy after neoadjuvant chemotherapy in cases of extensive breast malignancies [28].

Due to the lack of sufficient data available currently to endorse NAC as a standard procedure, there is a prospective role for SLNB following neoadjuvant chemotherapy on an individual basis.

\section{Conclusion}

SLNB nowadays has been recommended as a preferable treatment to ALND and also to avoid the use of ALND in a clinically negative axillary node. Three factors that will determine the ability to identify SLN are tumor palpability, surgeon's experience, and the biopsy 
technique. The tendencies of a nonpalpable tumor are greater toward the failure of identifying SLN; thus, in these cases, the dual-injection technique (with blue dye and radiocolloid) should be recommended. Despite the limitations of the dual technique in SLNB, further studies are currently being conducted to find a more effective method to detect the sentinel node.

\section{References}

1. American Cancer Society. Cancer Facts and Figures 2018. Atlanta: American Cancer Society; 2018.

2. Leong SP, Shen ZZ, Liu TJ, Agarwai G, Tajima T, Paik NS, et al. Is breast cancer the same disease in Asian and Western countries? World J Surg. 2010;34(10):2308-24. https://doi. org/10.1007/s00268-010-0683-1 PMid:20607258

3. Faries MB, Bedrosian I, Reynolds C, Nguyen HQ, Alavi A, Czerniecki BJ. Active macromolecule uptake by lymph node antigen-presenting cells: A novel mechanism in determining sentinel lymph node status. Ann Surg Oncol. 2000;7(2):98-105. https://doi.org/10.1007/s10434-000-0098-6

PMid: 10761787

4. Veronesi U, Adamus J, Bandiera DC, Brennhovd IO, Caceres E, Cascinelli N, et al. Inefficacy of immediate node dissection in stage 1 melanoma of the limbs. N Engl J Med. 1977;297(12):627-30. https://doi.org/10.1056/nejm197709222971202 PMid:895764

5. Krag DN, Weavert DL, Alex JC, Fairbank JT. Surgical resection and radiolocalization of the sentinel lymph node in breast cancer using a gamma probe. Surg Oncol. 1993;2(6):335-9. https://doi. org/10.1016/0960-7404(93)90064-6

PMid:8130940

6. Turkbey B, Ravizzini G, Choyke PL, Kobayashi H. Lymphangiogenesis and imaging of the lymphatics in cancer. In: Stacker SA, Achen MG, editors. Lymphangiogenesis in Cancer Metastasis Cancer Metastasis-biology and Treatment. Vol. 13. Switzerland: Springer; 2012. p.159-84. https://doi. org/10.1007/978-90-481-2247-9_7

7. Giuliano AE, Ballman KV, Mccall L, Beitsch PD, Brennan MB, Kelemen PR, et al. Effect of axillary dissection VS no axillary dissection on 10-year overall survival among women with invasive breast cancer and sentinel node metastasis the ACOSOG Z0011 (alliance) randomized clinical trial. JAMA. 2017;318(10):918-26. https://doi.org/10.1001/jama.2017.11470 PMid:28898379

8. Kreienberg R, Albert US, Folimann M, Kopp IB, Kuhn T, Wockel A. Interdisciplinary GoR level III guidelines for the diagnosis, treatment, and follow-up care of breast cancer. Geburtshilfe Frauenheilkd. 2013;73(6):556-83. https://doi. org/10.1055/s-0032-1328689

PMid:24771925

9. Ban EJ, Lee JS, Koo JS, Park S, Kim S I, Park B. Breast cancer how many sentinel lymph nodes are enough for accurate axillary staging in T1-2 breast cancer? J Breast Cancer. 2011;14(4):296300. https://doi.org/10.4048/jbc.2011.14.4.296 PMid:22323916

10. Gould EA, Winship T, Philbin PH, Kerr HH. Observations on a sentinel node in cancer of the parotid. Cancer. 1960;13:77-8. https://doi.org/10.1002/1097-0142(196001/02)13:1<77:aidcncr2820130114>3.0.co;2-d

\section{PMid: 13828575}

11. Wespes E, Simon J, Schulman C. Cabanas approach: Is sentinel lymph node biopsy reliable for staging penile carcinoma? Urology. 1986;28(4):278-9. https://doi. org/10.1016/0090-4295(86)90004-x

\section{PMid:3765235}

12. Cochran AJ, Wen D, Morton DL, Cochran AJ, Angeles L. Management of the regional lymph nodes in patients with cutaneous malignant melanoma. World J Surg. 1992;16(2):214-21. https://doi.org/10.1007/bf02071523 PMid:1561801

13. Giuliano AE, Kirgan DM, Guenther JM, Morton DL. Lymphatic mapping and sentinel lymphadenectomy for reast cancer. Ann Surg. 1994;220(3):391-8. https://doi. org/10.1097/00000658-199409000-00015

PMid:8092905

14. Krag DN, Anderson SJ, Julian TB, Brown AM, Harlow SP, Costantino JP, et al. Sentinel-lymph-node resection compared with conventional axillary-lymph-node dissection in clinically node-negative patients with breast cancer: Overall survival findings from the NSABP B-32 randomised phase 3 trial. Lancet Oncol. 2010;11(10):927-33. https://doi.org/10.1016/ s1470-2045(10)70207-2 PMid:20863759

15. James TA, Coffman AR, Chagpar AB, Frcs C, Boughey JC, Klimberg VS, et al. Troubleshooting sentinel lymph node biopsy in breast cancer surgery. Ann Surg Oncol. 2016;23(11):3459-66. https://doi.org/10.1245/s10434-016-5432-8

PMid:27444110

16. Bonneau C, Bendifallah S, Reyal F, Rossi L, Rouzier R. Association of the number of sentinel lymph nodes harvested with survival in breast cancer. Eur J Surg Oncol. 2015;41(1):52-8. https://doi.org/10.1016/j.ejso.2014.11.004

PMid:25466979

17. Morrow M, Rademaker AW, Bethke KP, Talamonti MS Dawes LG, Clauson J, et al. Learning sentinel node biopsy: Results of a prospective randomized trial of two techniques. Surgery. 1999;126(4):714-20. https://doi.org/10.1016/ s0039-6060(99)70127-3 PMid: 10520920

18. Stein RG, Fricker R, Rink T, Fitz H, Blasius S, Diessner J, et al. Evaluation of sentinel lymph node biopsy and axillary lymph node dissection for breast cancer treatment concepts-a retrospective study of 1,214 breast cancer patients. Breast Care (Basel). 2017;12(5):324-8. https://doi.org/10.1159/000477610 PMid:29234253

19. Pitsinis V, Provenzano E, Kaklamanis L, Wishart GC Benson JR. Indocyanine green fluorescence mapping for sentinel lymph node biopsy in early breast cancer. Surg Oncol. 2015;24(4):375-9. https://doi.org/10.1016/j.suronc.2015.10.002 PMid:26555151

20. Hojo T, Nagao T, Kikuyama M, Akashi S, Kinoshita T. Evaluation of sentinel node biopsy by combined fluorescence and dye method and lymph flow for breast cancer. Breast. 2010;19(3):210-3. https://doi.org/10.1016/j.breast.2010.01.014 PMid:20153649

21. Sugie T, Sawada T, Tagaya N, Kinoshita T, Yamagami K, Suwa H, et al. Comparison of the indocyanine green fluorescence and blue dye methods in detection of sentinel lymph node in earlystage breast cancer. Ann Surg Oncol. 2013;20(7):2213-8. https://doi.org/10.1245/s10434-013-2890-0 PMid:23429938

22. Van der Vorst JR, Schaafsma BE, Verbeek FP, Hutteman M, Mieog JS, Lowik CW, et al. Randomized comparison of near-infrared fluorescence imaging using indocyanine green 
and $99(m)$ technetium with or without patent blue for the sentinel lymph node procedure in breast cancer patients. Ann Surg Oncol. 2012;19(13):4104-11. https://doi.org/10.1245/ s10434-012-2466-4

PMid:22752379

23. Nielsen MA, Bull J, Culpan AM, Munyombwe T, Sharma N, Whitaker $\mathrm{M}$, et al. Preoperative sentinel lymph node identification, biopsy and localisation using contrast enhanced ultrasound (CEUS) in patients with breast cancer: A systematic review and meta-analysis. Clin Radiol. 2017;72(11):959-71. https://doi.org/10.1016/j.crad.2017.06.121

PMid:28774472

24. Colleoni M, Viale G, Zahrieh D, Pruneri G, Gentilini O, Veronesi P, et al. Chemotherapy is more effective in patients with breast cancer not expressing steroid hormone receptors: A study of preoperative treatment. Clin Cancer Res. 2004;10(19):6622-8. https://doi.org/10.1158/1078-0432.ccr-04-0380

PMid:15475452

25. van Deurzen $\mathrm{CH}$, Vriens BP, Tjan-Heijnen VC, van der Wall E, Albregts $M$, van Hilligersberg $R$, et al. Accuracy of sentinel node biopsy after neoadjuvant chemotherapy in breast cancer patients:Asystematicreview. EurJ Cancer. 2009;45(18):3124-30. https://doi.org/10.1016/j.ejca.2009.08.001

PMid:19716287

26. KuehnT, Bauerfeind I,FehmT,Fleige B, Hausschild M, Helms G, etal Sentinel-lymph-node biopsy in patients with breast cancer before and after neoadjuvant chemotherapy (SENTINA): A prospective, multicentre cohort study. Lancet Oncol. 2013;14(7):609-18. https:// doi.org/10.1016/s1470-2045(13)70166-9

PMid:23683750

27. Enokido K, Watanabe C, Nakamura S, Ogiya A, Osako T, Akiyama $F$, et al. Sentinel lymph node biopsy after neoadjuvant chemotherapy in patients with an initial diagnosis of cytologyproven lymph node-positive breast cancer. Clin Breast Cancer. 2016;16(4):299-304. https://doi.org/10.1016/j.clbc.2016.02.009 PMid:26993216

28. Classe JM, Bordes V, Campion L, Mignotte H, Dravet F, Leveque J, et al. Sentinel lymph node biopsy after neoadjuvant chemotherapy for advanced breast cancer: Results of ganglion sentinelle et chimiotherapie neoadjuvante, a french prospective multicentric study. J Clin Oncol. 2009;27(5):726-32. https://doi. org/10.1200/jco.2008.18.3228

PMid:19114697 